\title{
Cardiac Contusions
}

\author{
Brian Brewer $^{1}$ • Ben L. Zarzaur ${ }^{1}$
}

Published online: 5 October 2015

(C) Springer International Publishing AG 2015

\begin{abstract}
Cardiac contusion has been one of the most discussed topics in the trauma community. The discussions are centered around appropriate nomenclature, screening mechanisms, diagnostic modalities, and ultimately management principles. Controversy over nomenclature exists because cardiac contusion has been used to describe an array of injuries ranging from a transient arrhythmia to free rupture of the myocardial wall. The mainstays of screening have been electrocardiograms and cardiac isoenzymes. Although there is a substantial body of literature, few other screening or diagnostic modalities have been widely accepted. Echocardiography has gained favor as a confirmatory test and to determine presence or extinct of structural damage. Management has traditionally been supportive including volume repletion, correcting arrhythmias, and repairing structural damage. Recently, there has been some promising alternative treatments including transcatheter closure of ventricular septal defects, anti-inflammatory agents, and post-injury therapeutic hypothermia. Although screening and diagnostic mechanism have remained relatively unchanged, it appears that innovative management of severe cardiac contusions has gained some momentum over the recent years.
\end{abstract}

This article is part of the Topical Collection on Blunt Trauma to the Chest

Ben L. Zarzaur

bzarzaur@iupui.edu

Brian Brewer

briabrewe@iupui.edu

1 Department of Surgery, Indiana University School of Medicine, 1604 Capitol Avenue, Suite B241, Indianapolis, IN 46202, USA
Keywords Cardiac contusion · Blunt cardiac injury · Myocardial injury $\cdot$ Myocardial contusion $\cdot$ Blunt chest trauma $\cdot$ Chest trauma $\cdot$ Contusio cordis $\cdot$ Commotio cordis . Cardiac rupture

Studying cardiac injuries has been a tremendous challenge mainly due to diverse causes and variations in nomenclature and classifications. The mechanisms of injury include falls, motor vehicle crashes, crush injuries, assault, blast injuries, and iatrogenic causes such as those seen in CPR. Other mechanisms include direct energy transfer to the heart or by compression of the heart between the sternum and the vertebral column at the time of an accident.

\section{Introduction}

Cardiac contusion or contusio cordis was first described as early as 1763 as a result of a blow to the chest during an altercation at a public house. After the blow to the chest, the patient developed progressive signs of heart failure and he ultimately died of a sudden dysrhythmia. The diagnosis was confirmed by autopsy where a small bruise was found on the right ventricle of the patient. While our understanding of anatomy, physiology, and medicine has increased since 1763, our ability to definitively diagnose and treat cardiac contusions remains largely the same [1]. The gold standard for diagnosis is still autopsy and definitive treatment remains supportive.

\section{Incidence}

The true incidence of cardiac contusions is difficult to estimate due to the lack of a gold standard method of diagnosis other than autopsy or heart biopsy. We do know that cardiac trauma 
is identified in less than $10 \%$ of all trauma admissions in the National Trauma Data Bank, yet these injuries have a higher mortality than any other organ system [2]. A post-mortem study estimated the incidence of immediate fatalities from blunt chest trauma to be $17.3 \%$, with cardiac injuries being found in $11.9 \%$ of the cases [3]. Cardiac injuries are found to be a major contributor, $45-76 \%$, of the patients who die on the scene [3, 4]. A recent single center experience out of South Africa found the incidence of blunt cardiac injury in patients with blunt chest trauma to be $50 \%$; the most frequent mechanism being motor vehicle collisions (MVC) [5•].

Patients with CC who do survive to the hospital are complex because of the variability in presentation. This spectrum of injury makes the subject highly controversial. The documented estimates of the incidence of $\mathrm{CC}$ has a very wide range, from 0 to $76 \%$. A recent prospective study of 210 patients with blunt chest injury who received both transthoracic echocardiograms (TTEs) and electrocardiograms (ECGs) found that $23.4 \%$ had abnormal TTE and 16.2 \% also had an abnormal ECG. However, many of these patients were asymptomatic and remained so during observation [6]. These less severe cardiac injuries should be divided into two main groups: (1) structural cardiac injuries (intramural hematoma, rupture, valvular injury, etc.) and (2) electrical disturbances (atrial and ventricular dysrhythmias, conduction abnormalities) as suggested by Yousef et al. [2]. Documenting injuries using these classifications will allow us to obtain a better grasp on the true incidence of clinically significant blunt cardiac injuries. Unfortunately, it will have to suffice to know that severe chest trauma has a significant incidence of cardiac contusion and providers should have a high index of suspicion and take steps to rule out these potentially significant injuries [7].

\section{Clinical Manifestation and Classification}

Cardiac contusions (CCs) most often present as dysrhythmias on admission ECG [7]. The most common dysrhythmia is premature ventricular contractions. Other common electrical presentations include sinus tachycardia, ventricular tachycardia, supraventricular tachycardia, and ventricular fibrillation (Wall 2013) [7]. These injuries can also present as conduction disturbances. Right bundle branch block is the most frequently encountered conduction disturbance following trauma [8]. This presentation is not surprising given the relatively anterior location of the right-sided heart structures. Elevated biochemical markers and wall motion abnormalities on transesophageal echocardiography (TEE) have been used to diagnose subclinical CC [9•]. Hemodynamically stable patients presenting with signs of CC usually have transient findings that remain clinically insignificant [10]. Much of the controversy surrounding $\mathrm{CC}$ involves this category of patients. It has a been a priority to the trauma community to identify patients who require monitoring or further evaluation for $\mathrm{CC}$ but have no other associated injuries that require evaluation [9•].

More dramatic are the clinically significant $\mathrm{CC}$ that often present as overt heart failure demonstrated by hypotension or life-threatening dysrhythmias. Severe injuries can be associated with signs of other end organ dysfunction. These patients often present with a clinical picture of shock out of proportion to blood loss and what is to be expected from their other injuries [11, 12]. The most severe of these is CC with free chamber rupture. These injuries are largely fatal due to the rapid development of acute cardiac tamponade. According to Fulda et al., tamponade from chamber rupture is one of the most frequent causes of traumatic shock in patients with blunt chest trauma. It is imperative that clinicians have a high index of suspicion and recognize these injuries promptly [13]. Fortunately, clinically apparent blunt cardiac injuries are not as much of a dilemma because their clinical presentations direct the diagnostic modalities and management plans.

\section{Evaluation}

Screening for and diagnosing CC can be very challenging secondary to the relatively occult presentation of most of these injuries that survive to the hospital. The only Level 1 recommendation found in the Eastern Association for the Surgery of Trauma Guidelines (EAST) is that an electrocardiogram should be ordered on all patients who are suspected to have CC [9॰]. However, we have chosen to spend time critically reviewing the screening and diagnostic modalities because most of the recent literature attempts to tackle this challenging problem.

\section{ECG}

The single, most valuable screening tool for $\mathrm{BCI}$ is the ECG [14-16]. Nagy et al. has one of the most compelling prospective studies that determined patients sustaining blunt chest trauma that have a normal ECG, normal hemodynamics, and no dysrhythmias required no further intervention for CC [16]. This trend was confirmed in a study by Velmahos et al. with 333 subjects with blunt chest trauma, which demonstrated a normal ECG had a negative predictive value of $98 \%$ for significant CC [17]. Subsequently, the adequacy of ECG alone has been challenged by many studies suggesting that ECG must be used in combination with other modalities to definitively rule out $\mathrm{CC}$. Older literature advocates an admission ECG and a repeat ECG 8-24 h later. However, this simple algorithm can be resource intensive if all patients with suspicion for $\mathrm{CC}$ have to be monitored for $24 \mathrm{~h}$. The consensus now is that ECG is the single best predictor of CC but not adequate as a sole screening tool [9•, 17, 18]. This change is perhaps the most significant change since the last published practice management guidelines on $\mathrm{CC}$ by the Eastern Association for the Surgery of Trauma in 1998 [9•, 
19]. The majority of the recent literature has focused on determining a mechanism to adequately rule out $\mathrm{CC}$ in conjunction with or independent of ECG.

\section{Cardiac Enzymes}

Cardiac enzymes where initially believed to be unhelpful in screening for BCI [19-21]. This belief was partly related to the fact that mild elevations in the enzymes resulted in unnecessary, extensive workups to rule out cardiac disease. Multiple studies have shown that creatinine phosphokinase (CK) and creatinine phosphokinase-MB (CK-MB) are not useful in evaluating patients with blunt chest trauma [19, 22-24]. Similar to $\mathrm{CK}$ and CK-MB, troponin I levels were initially classified as unhelpful $[7,25,26]$. Bertinchant et al. concluded that troponins have improved specificity over CK and CKMB, but the sensitivity is low and they have low predictive value in diagnosing myocardial contusion [25]. Additional studies determined the sensitivity of troponin I in screening for BCI to be between 23 and $100 \%$ [25, 27]. However, the ability to isolate different isoforms of troponin I (Tn I), specifically the cardiac isoform (cTn I), has encouraged researchers to revisit the issue. Ferjani et al. found the specificity of these isoenzymes were improved over whole troponin 1 , but the sensitivity was unacceptably low at $31 \%$ [28]. A specificity of $91 \%$ was attained by increasing the positive troponin $\mathrm{T}$ threshold to $0.5 \mathrm{ug} / \mathrm{L}$ [29]. A commonly employed use of cardiac enzymes in screening for CC is troponin I in combination with ECG findings. Some studies have demonstrated that a combination of abnormal ECG findings and abnormal troponin I levels has a $100 \%$ sensitivity for the detection of clinically significant blunt chest trauma [18]. More importantly, it is widely accepted that normal ECG findings combined with normal troponin I levels can adequately rule out clinically significant $\mathrm{CC}$ and patients do not warrant any further work-up or monitoring [17, 27, 29].

\section{Echocardiography}

Transthoracic echocardiography (TTE) has not been widely recommended for routine evaluation for patients suspected to be at risk for CC. Although useful in the fast exam to evaluate for pericardial effusion, an increased skillset is required to interpret the subtle findings that may be associated with CC. Another major limitation is the poor images obtained when patients have severe chest wall or thoracic trauma that can limit the ability to detect CC $[30,31]$. 'Transesophageal echocardiography (TEE) may offer better acquisition of images and be more useful in the evaluation patients suspected of CC but it has not been widely employed for screening given the resources required and relative invasiveness of the exam. The literature supports reserving TTE and TEE for patients with clinical findings suggestive of CC [17, 23, 24]. TEE may be helpful in offering information such as cardiac wall abnormalities, cardiac tamponade, thrombus, and shunting. Skinner et al. found that $79 \%$ of those who met their indication for TEE had abnormal findings on the study [5•]. Current literature suggests echocardiogram is a useful adjunct to when evaluating hypotensive patients and patients with other clinical signs including pain, ECG abnormalities, and elevated enzymes [32••].

\section{Computed Tomography and Magnetic Resonance Imaging}

Historically, helical chest CT has been deemed inadequate for the evaluation of CC $[19,33]$. The recent use of multi-detector CT scan (MDCT) with CT-angiography capabilities has resulted in some encouraging data. MDCT has been shown to be highly sensitive in blunt chest trauma and to be helpful in the characterization of blunt thoracic injuries [33-37]. A prospective study by Shiekh et al. found that 64 MDCT was $92 \%$ sensitivity and $98 \%$ specificity in detecting coronary artery disease [38]. The rapid evaluation that this modality offers could be beneficial in distinguishing myocardial contusion from peri-traumatic myocardial infarction and dictate which patients deserve extensive cardiac work-up [9]. Magnetic resonance imaging (MRI) is of interest for the same reasons as MDCT. There is some evidence that contrast-enhanced cardiac MRI can be helpful in differentiating $\mathrm{CC}$ from acute peritraumatic myocardial infarction $[39,40]$. Pericardial injury wall abnormalities and valvular abnormalities can be detected. MRI usefulness may be limited because these studies are not suitable for hemodynamically unstable patients or the critically injured. Although there are many case reports and small series regarding MRI and CT, there is no level I evidence to support the adequacy of these studies as a screening too [9•].

\section{Treatment}

Complete free wall rupture is the most dramatic of all blunt cardiac injuries. These injuries are rarely encountered in the hospital setting due to the high immediate mortality. Those who make it to the hospital generally present with cardiac tamponade. The pericardium acts as a wall and the injuries are essentially a pseudoaneurysm. Occasionally, these patients can present up to 2 weeks later with a delayed rupture of the wall [41]. The priority in this situation is to relieve the tamponade physiology and repair the heart. In emergent circumstances, these injuries can be temporized using a Foley catheter and stapling has also been described [7]. Definitively, sutures can be used in a horizontal mattress type fashion taking big bites through the myocardium. Choice of suture material can vary. The authors have found that large needles and braided non-absorbable sutures are easier to work with in emergent situations. Care should be taken not to include the major coronary vessels, if possible. Atrial and septal ruptures are generally less dramatic and sometimes present with murmur or evidence of shunting. Other structural damages diagnosed by ECHO or 
MDCT warrant the consultation of a cardiac surgeon. Traumatic VSD has been reported and traditionally requires an operation with patch closure, although transcatheter closures seems promising for these rare injuries [40, 42]. Given the advancements in minimally invasive thoracic surgery, a specialist's opinion should be sought when appropriate.

Dysrhythmias are the most commonly encountered presentation of CC [42]. Current management is limited to volume repletion, vasopressors, and anti-arrhythmic agents. Currently, there are attempts to apply some of the principles used to manage other forms of myocardial injury (ischemia, cardiac surgery) to CC. Data from an animal study by Demir et al. is encouraging demonstrating that treatment with methylprednisolone and quercetin may be beneficial in CC [43]. Rat models with $\mathrm{CC}$ had a significant reduction in Tn-I and TNF-alpha levels at 7 days, when treated with a combination of methylprednisolone and quercetin, compared to those without treatment. The treated animals were also found to have a reduction in their histopathological degeneration and necrosis scores. A case report by Carlson et al. suggest that a patient with commotio cordis had a improved outcome when therapeutic hypothermia protocol was instituted after an arrest secondary to a direct blow to the heart [44]. This victim with at least $15 \mathrm{~min}$ of ventricular fibrillation arrest and a Glasgow Coma Scale (GCS) score of $5 \mathrm{~T}$. The patient was ultimately discharged with an improved GCS of 14 . These new innovative management modalities are potentially promising.

\section{Conclusion}

Blunt cardiac injury remains a controversial topic in the field of trauma due to variation in terminology and the range of injuries that term encompasses. There has been little advancement of the literature in the past 5 years. Current literature is mostly centered around screening for these injuries and adequately ruling out these injuries. The current sentiment is that extensive screening in hemodynamically normal patients with normal initial ECG is unnecessary and costly. Perhaps the most notable change is the EAST changed its practice management guidelines to recommend troponin I levels in addition to ECG to rule out CC. These changes have been helpful, to avoid costly workups in patients with other injuries that were thought to be associated with blunt cardiac injury such as sternal fractures. ECHO continues to be valuable in diagnosing specific blunt cardiac injuries but has not proven to be practical for routine screening. CT and MRI technology is continuously advancing in speed and detail and will likely become helpful as a screening and as a means of differentiating the etiology of cardiac symptoms. Advances in minimally invasive treatment modalities are numerous and need to be studied more in the setting of trauma.

Compliance with Ethics Guidelines
Conflict of Interest Drs. Brewer and Zarzaur declare that they have no conflicts of interest.

Human and Animal Rights and Informed Consent This article does not contain any studies with human or animal subjects performed by any of the authors.

\section{References}

Papers of particular interest, published recently, have been highlighted as:

- Of importance

•- Of major importance

1. Akenside M. An account of a blow upon the heart, and of its, effects: by Mark Akenside, M. D. F. S. and physician to Her majesty. Philos Trans. 1973;53:353-5.

2. Yousef R, Carr JA. Blunt cardiac trauma: a review of current knowledge and management. Ann Thorac Surg. 2014;98:1134-40.

3. Turan AA, Karayel FA, Akyildiz E, et al. Cardiac injuries caused by blunt trauma: an autopsy based assessment of the injury pattern. $\mathrm{J}$ Forensic Sci. 2010;55:82-4.

4. Turk EE, Tsang YW, Champaneri A, Pueschel K, et al. Cardiac Injuries in car occupants in fatal motor vehicle collisions- an autopsy based study. J Forensic Leg Med. 2010;17:339-43.

5. Skinner DL, Laing GL, Rodseth RN, et al. Blunt cardiac injury in critically ill trauma patients: a single centre experience. Injury. 2015;46(1):66-70. Reiterates the usefulness of serum troponins in patients with high index of suspicion for $\mathrm{CC}$, and recommends TTE for those with CC diagnosis to rule out cardiac structural injury.

6. Bahar AM, Nouri M, Alizadeh L, Namvar F, Asadi M. Estimated incidence of cardiac contusion using transthoracic echocardiography in patients suffering from severe blunt trauma to the chest. Acta Chir Belg. 2014;114(2):105-9.

7. Wall MJ, Tsai PI, Mattox KL. Heart and thoracic vascular injuries. In: Moore EE, Mattox KL, Feliciano DV, editors. Trauma. 7th ed. New York: McGraw-Hill; 2013.

8. Berk WA. ECG findings in non-penetrating chest trauma: a review. J Emerg Med. 1987;5:209-15.

9. Clancy K, Velopulos C, Bilaniuk J, et al. Screening for blunt cardiac injury: an eastern association for the surgery of trauma practice management guideline. J Trauma. 2012;73(5):S301-6. Solidifies ECG as screening tool for $\mathrm{CC}$ and added troponin $\mathrm{I}$ as part of the recommended screening tools.

10. Gunnar WP, Martin M, Smith RF, et al. The utility of cardiac evaluation in the hemodynamically stable patient with suspected myocardial contusion. Am Surg. 1991;578:373-7.

11. Leavitt BJ, Meyer JA, Morton JR, et al. Survival following nonpenetrating traumatic rupture of the cardiac chambers. Ann Thorac Surg. 1987;44:532-5.

12. Brathwaite CE, Rodriquez A, Turney SZ, et al. Blunt traumatic cardiac rupture. A 5-year experience. Ann Surg. 1990;212:701-4.

13. Fulda G, Brathwaite CE, Rodriguez A, et al. Blunt traumatic rupture of the heart and pericardium: a ten-year experience (19791989). J Trauma. 1991;31:167-72.

14. Illig KA, Swierzewski MJ, Feliciano DV, et al. A rationale screening and treatment strategy based on electrocardiogram alone for suspected cardiac contusion. Am J Surg. 1991;162:537-44.

15. Fildes JJ, Betlej TM, Manglano R, et al. Limiting cardiac evaluation in patients with suspected myocardial contusion. Am Surg. 1995;61:832-5. 
16. Nagy K, Krosner S, Robert R, et al. Determining which patients require evaluation for blunt cardiac injury following blunt chest trauma. World J Surg. 2001;25:108-11.

17. Velmahos G, Karaiskakis M, Salim A, et al. Normal electrocardiography and serum troponin I levels preclude the presence of clinically significant blunt cardiac injury. J Trauma. 2003;54:45-51.

18. Salim A, Velmahos G, Jindal A, et al. Clinically significant blunt cardiac trauma: role of serum troponin levels combined with electrocardiographic findings. J Trauma. 2001;50:237-43.

19. Pasquale M, Nagy K, Clarke J. Practice management guideline for screening of blunt cardiac injury. J Trauma. 1998;44:941-56.

20. Biffl WL, Moore FA, Moore EE, et al. Cardiac enzymes are irrelevant in the patient with suspected myocardial contusion. Am J Surg. 1993;168(6):523-7. discussion 527-8.

21. Riezzo I, Pomara C, Neri M, Rossi G, et al. Cardiac contusion: ending myocardial confusion in this capricious syndrome. Int $\mathrm{J}$ Cardiol. 2008;128(3):e107-10.

22. Swaanenburg J, Klaase J, DeJongste M, et al. Troponin I, troponin T, CKMB-activity and CKMB- mass as markers for detection of myocardial contusion in patients who experienced blunt trauma. Clin Chim Acta. 1998;272:171-81.

23. Fulda G, Giberson F, Hailstone D, et al. An evaluation of serum troponin $\mathrm{T}$ and signal-averaged electrocardiography in predicting electrocardiographic abnormalities after blunt chest trauma. J Trauma. 1997;43:304-12.

24. Garcia-Fernandez M, Lopez-Perez J, Perez-Castellano N, et al. Role of transesophageal echocardiography in the assessment of patients with blunt chest trauma: correlation of echocardiographic findings with the electrocardiogram and creatinine kinase monoclonal antibody measurements. Am Heart J. 1998;135:476-81.

25. Bertinchant JP, Polge A, Mohty D, et al. Evaluation of incidence, clinical significance, and prognostic value of circulating cardiac troponin I and T elevation in hemodynamically stable patients with suspected myocardial contusions after blunt chest trauma. J Trauma. 2000;48(5):924-31.

26. Collins J, Cole F, Weireter L, et al. The usefulness of serum troponin $\mathrm{T}$ and signal-averaged electrocardiography in evaluating cardiac injury. Am Surg. 2001;67:821-6.

27. Adams III J, Davilla-Roman V, Bessey P, et al. Improved detection of cardiac contusion with cardiac troponin I. Am Heart J. 1996;131: 308-12.

28. Ferjani M, Droc G, Dreux S, et al. Circulating cardiac troponin T in myocardial contusion. Chest. 1997;111:427-33.

29. EL-Chami MF, Nicholson W, Helmy T. Blunt cardiac trauma. J Emerg Med. 2008;35(2):127-33.

30. Chirillo F, Totis O, Cavarzerani A, et al. Usefulness of transthoracic and transesophageal echocardiography in recognition and management of cardiovascular injuries after blunt chest trauma. Heart. 1996;75:301-6.

31. Oh JK, Seward JB, Khanderia BK, et al. Transesophageal echocardiography in critically ill patients. Am J Cardiol. 1990;66:1492-5.

32.• Ferrada P, Vanguri P, Anand RJ, et al. A, B, C, B, echo: limited transthoracic echocardiogram is a useful tool to guide therapy for hypotension in the trauma bay- a pilot study. J Trauma Acute Care Surg. 2013;74(1):220-3. Evaluates the utility of limited thoracic echo for unstable trauma patients in the trauma bay; found that LTTE changed the therapy in $65 \%$ of the patients greater than 65 years.

33. Vignon P, Boncoeur M, Francois B, et al. Comparison of multiplane transesophageal echocardiography and contrast-enhanced helical $\mathrm{CT}$ in the diagnosis of blunt traumatic cardiovascular injuries. Anesthesiology. 2001;94:615-22.

34. Scaglione M, Pinto A, Pedrosa L, et al. Multidetector row computed tomography and blunt chest trauma. Eur J Radiol. 2012;65:377-88.

35. Oikonomou A, Prassopoulos P. CT imagine of blunt chest trauma. Insights Imaging. 2011;2:281-95.

36. Mirvis SE. Imagine of acute thoracic injury: the advent of MDCT screening. Semin Ultrasound CT MR. 2005;26:305-31.

37. Co SJ, Yong-Hing CJ, Galea-Soler S, et al. Role of imaging in penetrating and blunt traumatic injury to the heart. Radiographics. 2011;31:E101-5.

38. Sheikh M, Ben-Nakhi A, Shukkur AM, et al. Accuracy of 64multidetector-row computed tomography in the diagnosis of coronary artery disease. Med Princ Pract. 2009;18:323-8.

39. Mabranque G, Serfaty JM, Himbert D, et al. Myocardial infarction after blunt chest trauma: usefulness of cardiac ECG-gated CT and MRI for positive and aetiologic diagnosis. Emerg Radiol. 2011;18: 271-4.

40. Southam S, Jutila G, Ketai L. Contrast-enhanced cardiac MRI in blunt chest trauma: differentiating cardiac contusion from acute peri-traumatic myocardial infarction. J Thorac Imaging. 2006;21: 176-8.

41. Rodriquez A, Ong A. Delayed rupture of a left ventricular aneurysm after blunt trauma. Am Surg. 2005;71:250-1.

42. Kasem M, Kanthimathinathan HK, Mehta C, et al. Transcatheter device closure of a traumatic ventricular septal defect. Ann Pediatr Cardiol. 2014;7(1):41-4.

43. Demir F, Güzel A, Kat C, Karadeniz C, Akdemir U, Okuyucu A, et al. A combination of methylprednisolone and quercetin is effective for the treatment of cardiac contusion following blunt chest trauma. Braz J Med Biol Res. 2014;47(9):766-72.

44. Carlson DW, Pearson RD, Haggety PF, Strilka RJ, Abella BS, Gourley PE. Commotio cordis, therapeutic hypothermia, and evacuation from a United States military base in Iraq. J Emerg Med. 2013;44(3):620-4. 